\title{
Coding Client Language in Motivational Interviewing for HIV Medication Adherence Using Self-Determination Theory
}

\author{
Ailbhe Hogan $^{1} \cdot$ Delwyn Catley ${ }^{2,3} \cdot$ Kathy Goggin ${ }^{4,5} \cdot$ Michael Evangeli $^{1}$
}

Published online: 23 January 2019

(C) The Author(s) 2019

\begin{abstract}
Background Both motivational interviewing (MI) and self-determination theory (SDT) emphasise the importance of an individual's autonomy. SDT proposes that motivation is on a continuum with autonomous motivation (AM) at the self-determined end of the spectrum. Whether client speech reflects AM is not coded in MI process studies, however, as it is subsumed under the broader category of change talk (CT). We aimed to code naturalistic speech within MI sessions for HIV medication (antiretroviral) adherence according to whether expressed motivation was autonomous or controlled. We then assessed relationships between adherence and both autonomous/controlled motivational speech $(\mathrm{AM} / \mathrm{CM})$ and CT.

Methods We developed a new coding tool (the SDT coding system: SDTCS) to measure naturally occurring AM speech and CM speech expressed during an MI session targeting antiretroviral (ART) adherence with 62 adults living with HIV (16 female; mean age 40 years). We coded the same sessions using the motivational interviewing skills code (MISC) 2.5 and then examined relationships with on-time ART adherence.

Results The SDTCS was developed using a combined inductive and deductive approach. Adequate reliability estimates were achieved and the measure possessed good content validity. Naturally occurring AM speech had a stronger relationship to ART adherence in the week after the MI session than CM speech. There was also some evidence that the relationship between AM speech and adherence was stronger than between CT and adherence.

Conclusion Future refinement and extension of the SDTCS could allow for a more nuanced understanding of client motivational speech that is currently provided by existing coding tools.
\end{abstract}

Keywords HIV $\cdot$ Adherence $\cdot$ Motivational interviewing $\cdot$ Self-determination theory

\section{Introduction}

Motivational interviewing (MI) is a goal-focused, personcentred counselling style that aims to elicit and strengthen the client's own motivation for behaviour change [1].

Michael Evangeli

michael.evangeli@rhul.ac.uk

1 Department of Psychology, Royal Holloway, University of London, Egham, Surrey TW20 0EX, UK

2 Center for Children's Healthy Lifestyles and Nutrition, Children's Mercy Kansas City, Kansas City, USA

3 Department of Pediatrics, University of Missouri - Kansas City, Kansas City, USA

4 Health Services and Outcomes Research, Children's Mercy Kansas City, Kansas City, USA

5 Schools of Medicine and Pharmacy, University of Missouri - Kansas City, Kansas City, USA
Understanding the active ingredients of MI will allow for appropriate adaptations of this intervention and may improve clinical outcomes, training, practice, and research [2].

Psycholinguistic analyses of MI sessions form the basis of MI process research studies, with MI theorists highlighting the importance of the language clients use about behaviour change $[3,4]$. Change talk (CT) is thought to be a key treatment mediator and is associated with behaviour change across diverse populations and behaviours [5, 6]. CT encompasses language describing the desire, ability, reasons, needs, and commitment to change. However, a recent meta-analysis failed to find a relationship between $\mathrm{CT}$ and outcome [7]. The absence of an effect at an aggregate level warrants further detailed examination of CT.

Self-determination theory [8] is proposed as a theoretical lens to understand how MI works [9]. SDT proposes that motivation is on a continuum from fully self-determined to non-self-determined action. The more self-determined a behaviour, the more likely a person is to initiate and persist with 
it. Autonomous motivation (AM) lies at the self-determined end of the spectrum where the behaviour has been chosen. Controlled motivation (CM) refers to engaging in behaviours for externally referenced reasons such as perceived coercion by others, to gain rewards or approval from others or to avoid feelings of guilt or increase feelings of self-worth. AM has been linked to engagement with and completion of alcohol treatment [10], tobacco abstinence [11], and adherence to medication [12], including HIV antiretroviral (ART) medication [13].

Change talk coding tools such as the motivational interviewing skills code [MISC] 2.5 [14] classify CT statements into separate categories (e.g., reasons or commitment for making a change) that do not distinguish the degree of selfdetermination. This may obscure an important dimension of change talk critical to its impact on behaviour change. To date, there has been no consideration of whether CT reflects apparent AM or CM. A method of classifying CT as likely reflecting AM or CM may enhance its predictive value.

$\mathrm{AM}$ and $\mathrm{CM}$ are usually measured through self-report questionnaires such as the Treatment Self-Regulation Questionnaire (TSRQ) [15]. While these measures are quick to administer, they possess limitations for examining psychotherapy mechanisms of change. For example, self-report measures are often developed deductively and refined using classical test theory, with data not fitting pre-existing theory discarded. This may limit their content validity [16]. In addition, and most importantly for MI process research, they are not useful in the analysis of client speech during sessions.

Given these limitations, and the varied evidence linking CT and behaviour change, we aimed to develop a novel method of measuring AM speech and CM speech from client speech in MI sessions from an ART adherence trial. We coded all speech within a session corresponding to these categories. We predicted that higher levels of ART adherence would be more closely associated with AM than CM speech. We also explored whether AM speech was more closely related to ART adherence than the broader, traditional MI category of CT. This reflects the fact that MI emphasises the effect of the amount, intensity, and sequence of change talk, whereas SDT focuses on the quality of change talk [17].

\section{Methods}

This was a secondary analysis of de-identified data collected as part of Project MOTIV8, an RCT exploring the use of MI to increase ART adherence [18]. Ethical approval for the study was first obtained from Institutional Review Boards in 2004.

\section{MOTIV8 Participants and MI Sessions}

Participants were recruited from six government-funded primary care outpatient clinics in Kansas City. Eligible participants were HIV positive, over the age of 18, English-speaking, and taking ART medication. Demographic and clinical information are presented in Table 1.

Individual face to face MI sessions were scheduled at baseline, weeks 1, 2, 6, 11, and 23. Participants also received four telephone sessions (weeks 4, 9, 15, and 19). Sessions lasted, on average, $25 \mathrm{~min}$. The baseline session consisted of information on adherence. Subsequent sessions used one of 11 skills-building modules (e.g., self-monitoring, goal setting). MI for motivation enhancement was the focus of the week 1 therapy session. MI sessions were provided by Master's degree level professionals, trained and supervised weekly by an expert MI trainer (second author).

Table 1 Participant demographic and clinical information $(n=62)$

\begin{tabular}{|c|c|c|}
\hline \multirow[b]{2}{*}{ Variable } & \multicolumn{2}{|l|}{ All participants } \\
\hline & Mean (SD) & Number (\%) \\
\hline Age, years & $40.1(10.1)$ & \\
\hline Male gender at birth & & $46(74.2)$ \\
\hline \multicolumn{3}{|l|}{ Ethnicity } \\
\hline African-American & & $33(53.2)$ \\
\hline White & & $23(37.1)$ \\
\hline Mixed & & $5(8.1)$ \\
\hline Other & & $1(1.6)$ \\
\hline \multicolumn{3}{|l|}{ Education } \\
\hline High School Degree or Less & & $31(50.0)$ \\
\hline More than High School Degree & & $31(50.0)$ \\
\hline \multicolumn{3}{|l|}{ Sexual orientation } \\
\hline Heterosexual & & $27(43.5)$ \\
\hline Homosexual & & $26(41.9)$ \\
\hline Bisexual & & $6(9.7)$ \\
\hline Other & & $1(1.6)$ \\
\hline Choose not to answer & & $2(3.2)$ \\
\hline \multicolumn{3}{|l|}{ Employment } \\
\hline Full-time & & $9(14.5)$ \\
\hline Part-time & & $6(9.7)$ \\
\hline Not currently employed & & $47(75.8)$ \\
\hline \multicolumn{3}{|l|}{ Depressive symptoms ${ }^{1}$} \\
\hline Above clinical threshold & & $36(58.1)$ \\
\hline First time taking ART & & $18(29.0)$ \\
\hline \multicolumn{3}{|c|}{ Viral load (copies/ml) _ baseline $(n=53)$} \\
\hline$<400$ & & $53(100)$ \\
\hline$\geq 400$ & & $0(0)$ \\
\hline CD4 count $\left(\right.$ cells $\left./ \mathrm{mm}^{3}\right)$ - baseline & $264.1(177.7)$ & \\
\hline
\end{tabular}

${ }^{1}$ Center for epidemiological studies depression scale (Radloff, 1977) 


\section{Sample Selection}

One hundred thirty-nine participants were randomised to receive the MI intervention in the MOTIV8 RCT. This study focused on the week 1 therapy session (MI for motivation enhancement) as this was delivered to all participants receiving MI. Due to the ceiling effect noted in the RCT data whereby participants reported high motivation to adhere to ART, this study focused on those participants with lower baseline adherence motivation (mean motivation to adhere $<10, n=$ 65). Ten participants with high baseline motivation to adhere (mean motivation to adhere $=10$ ) were randomly selected to achieve a sample of 75 sessions. Of this identified sample, two audio files were missing and in one session, the client spoke in both Spanish and English throughout. Therefore, these three participants were excluded. Of the 72 remaining sessions, a convenience sample of 66 sessions was coded and deemed sufficient for the purposes of the study. During data analysis, it was found that four participants had missing adherence data and they were excluded from the study leaving a final sample of $n=62$. Twelve additional sessions were randomly selected for development of the SDT coding system from the sessions not selected for the main analysis. All transcripts were parsed and coded by the first author.

\section{Measures}

\section{ART Adherence}

ART adherence data was collected using an electronic pill-cap (Medication Events Monitoring System [MEMS] cap), which captured the date and time when a medication bottle was opened. ART adherence was calculated as the percentage of prescribed ART doses taken on time (within $2 \mathrm{~h}$ either side of the scheduled dose time). Data were calculated at three separate intervals: (1) week 1 (the 7-day period before the first MI session); (2) week 2 (the 7-day period after the first MI session); and week 12 (30 days of adherence data prior to the 12 th week of the trial).

\section{Motivation to Adhere}

A brief self-report measure capturing baseline ART adherence motivation was devised. Participants rated from $0=$ not at all to $10=$ extremely, their need, reasons, readiness, and commitment to adhere strictly to the ART schedule $(\alpha=0.83)$.

\section{Motivational Interviewing Skills Code (MISC) 2.5}

Therapy sessions were coded using the MISC 2.5 [14] with twelve sessions (19\%) randomly selected for double coding by an undergraduate researcher. Training followed the procedures outlined in Moyers and Martin [19]. The first author spent $30 \mathrm{~h}$ of professional training in the use of the MISC 2.5 while an undergraduate researcher spent $15 \mathrm{~h}$ in training. The session was parsed into separate speech utterances and a behavioural code was assigned to each one. $\mathrm{CT}$ categories are as follows: desire (e.g., "I want to take medication every day"), ability (e.g., "I can stop missing doses"), reason (e.g., "Life will be better if I take my medication"), need (e.g., "I need to stop skipping my medication"), taking steps (e.g., "Last week I set an alarm to remind myself to take the meds"), commitment (e.g., "I'm going to start taking the medication tomorrow"), and other (i.e., change talk not captured by the other categories). To prevent coding drift, weekly coding meetings between the first author and the undergraduate researcher were held after every three sessions. The final author resolved any outstanding disagreements. Cohen's kappa [20] tested CT inter-rater agreement. There was substantial agreement between raters $(\mathrm{kappa}=0.69)$.

\section{SDT Coding System (SDT Coding System: SDTCS)}

A coding system was developed to quantify naturally occurring $\mathrm{AM}$ and $\mathrm{CM}$ speech. Eight of the transcripts selected for coding development were reviewed to identify all references to clients' motivation to adhere to ART. These adherence reasons were categorised as being either AM speech (e.g., "Taking my ART is important to me", "I take my meds cause it's just who I am.", "I take medication as I value the benefits I get from it") or CM speech (e.g., "My family/friends/doctor make me take it", "It makes my loved ones happy when I take my medication”, "I feel guilty if I don't comply with my medication regime") and were used as examples in the coding manual. Additional examples were generated following a review of the literature and existing self-report measures of AM and CM. Feedback was sought from one female and one male HIV service user recruited from the UK Children's HIV Association (CHIVA), a charity for children and young adults living with HIV. These service users were over the age of 18 , living with HIV and currently taking ART. The manual was piloted on the four remaining transcripts selected for coding development. Codes were agreed between the first author (a doctoral clinical psychology student) and final author (a qualified clinical psychologist) to produce gold-standard transcripts for training. The manual was further refined and extended based on coding meetings following this piloting process. The final SDTCS manual required the coding of all parsed client speech (parsed using the MISC 2.5) [14] as either AM speech, CM speech, or non-motivational client speech. All client speech which could not be coded as AM or CM speech was coded as non-motivational client speech and accounted for the majority of client speech. This category included what SDT would term "amotivational speech", which refers to the absence of any intention and thus motivation to engage in behaviour. Coding a statement as a "reason" 
to engage in a behaviour in the MISC 2.5 is time-specific. Therefore, only statements that related to current or very recent motivation to adhere were coded as AM or CM speech. All references to historical motivation were coded as nonmotivational client speech.

\section{Coding Training}

An undergraduate researcher (different individual to second coder for MISC coding) received $10 \mathrm{~h}$ of training in the SDTCS. This involved reading key SDT literature, one-toone teaching on using the coding manual, and practice coding using the gold-standard transcripts.

\section{Reliability-Testing}

Fifteen sessions (24\%) were randomly selected for double coding by the first author and undergraduate researcher. To prevent coding drift, weekly coding meetings were held after every five sessions coded. Consultation was provided by the final author for any outstanding disagreements.

\section{Data Analyses}

Analysis used SPSS 21. As one code (non-motivational client speech) accounted for the majority of client speech (87\%) inter-coder agreement for the SDTCS was assessed using Gwet's [21] AC1 statistic which has been developed to overcome this prevalence problem [22]. Pearson's correlations tested the relationships between ART adherence before (week 1) and after the MI session (weeks 2 and 12) and (a) AM speech and CM speech (b) CT. Bootstrapped confidence intervals were used due to skewed data. Follow-up tests for dependent correlations investigated if the correlations between AM speech and adherence, and CM speech and adherence, were significantly different from one another. The correlation coefficients were converted to $z$ scores using Fischer's transformation and established equations were applied to compute the asymptotic covariance of the estimates. Then an asymptotic $z$ test was performed [23].

\section{Results}

The overall inter-rater reliability of the SDTCS between the first author and undergraduate researcher, using Gwet's AC1 statistic, was 0.89 (near perfect agreement). Gwet's AC1 statistic of 0.95 (perfect agreement) was achieved for nonmotivational client speech, 0.45 (moderate agreement) for CM speech and 0.32 (fair agreement) for AM speech. Due to the lower reliabilities for AM speech and CM speech, all categorizations were verified by the final author before a final coding decision was made.

Table 2 displays the descriptive statistics for each variable.

Across the three time points for ART adherence data the correlations were larger for AM speech than for CT, which in turn were larger than for CM speech (see Table 3).

The relationships between CM speech and ART adherence were not significant at any of the time points. The relationships between AM speech and ART adherence at weeks 1 and 12 were not significant but there was a medium effect sized significant positive correlation between AM speech and ART adherence in the week following the MI session (week 2), $r$ $(60)=0.28, p<0.05,95 \%$ BCa CI $[0.06,0.45]$.

The association between AM speech and ART adherence after the MI session (week 2) was significantly larger than between CM speech and week 2 ART adherence; $z=2.37$, two-tailed, $p=0.02$. The association between AM speech and ART adherence was not significantly larger than the association between CM speech and ART adherence at either week $1(z=0.51$, two-tailed, $p=0.61)$ or week $12(z=1.03$, twotailed, $p=0.30)$.

\section{Discussion}

We aimed to (a) quantify AM speech and CM speech, as well as CT, through coding client speech during MI sessions and (b) investigate their relationships with ART adherence. This study is the first to attempt to measure within-session AM and CM speech. We were able to develop a coding scheme that achieved good inter-rater agreement across categories and fair to moderate agreement for SDT-specific categories. The reliability estimates
Table 2 Descriptive statistics of coding and adherence data

\begin{tabular}{lllll}
\hline Variable & Median & Interquartile range & Mean & SD \\
\hline Week 1 (\% doses taken on time) & 85.71 & $62.50-100.00$ & 76.05 & 29.85 \\
Week 2 (\% doses taken on time) & 89.29 & $57.14-100.00$ & 74.25 & 33.49 \\
Week 12 (\% doses taken on time) & 78.57 & $53.00-95.21$ & 69.42 & 29.38 \\
Autonomous motivation speech (count) & 3.00 & $1.75-6.00$ & 4.26 & 3.94 \\
Controlled motivation speech (count) & 6.50 & $4.00-10.00$ & 8.48 & 8.55 \\
Change talk (count) & 38.00 & $30.75-55.75$ & 45.87 & 24.69 \\
\hline
\end{tabular}


Table 3 Pearson's correlation coefficients $(r)$ between ART adherence (weeks 1,2,12) and autonomous and controlled motivation speech and change talk $(n=57)$

\begin{tabular}{llrr}
\hline Variable & $\begin{array}{l}\text { Autonomous } \\
\text { motivation speech }\end{array}$ & \multicolumn{1}{l}{$\begin{array}{l}\text { Controlled } \\
\text { motivation speech }\end{array}$} & Change talk \\
\hline Week 1 (\% of doses taken on time) & $0.09[-0.12,0.26]$ & $-0.01[-0.29,0.35]$ & $0.01[-0.27,0.32]$ \\
Week 2 (\% of doses taken on time) & $0.28^{*}[0.08,0.44]$ & $-0.07[-0.34,0.27]$ & $0.03[-0.21,0.30]$ \\
Week 12 (\% of doses taken on time) & $0.19[-0.04,0.38]$ & $0.03[-0.21,0.20]$ & $0.09[-0.09,0.30]$ \\
\hline
\end{tabular}

Note: $\mathrm{BCa} 95 \%$ confidence intervals in parentheses below each $r$ value $* p<0.05$

at the subscale level are in line with a reliability study of the MISC which found that $43 \%$ of the subscales achieved poor reliability estimates $(-0.03-0.36)$ and these were mainly low-frequency categories [24]. Our study represents a promising first attempt at testing SDT's theory of how motivation is associated with behaviour during the therapeutic process.

We predicted that AM speech would be more closely related to ART adherence than CM speech. The results offer tentative support for this prediction as there was a significant medium effect sized relationship between AM speech and ART adherence in the week following the MI session (week 2) and there was a non-significant positive association between ART adherence at the same time point and CM speech (with the former correlation being significantly larger than the latter). These results are in line with research showing that self-reported AM is related to successful medication adherence $[12,13]$ and with research showing that self-reported AM is better than $\mathrm{CM}$ at predicting behaviour change [10].

It is also promising that a medium effect size was detected in the relationship between AM speech and ART adherence given that a previous study using the TSRQ was only able to find a small effect $(r=0.15)$ between AM and ART adherence [13]. Although we found no significant relationship between either AM or CM speech and ART adherence at week 1 (before the coded MI session) and week 12, these findings likely reflect AM speech levels changing as a result of the MI session and the effects of these changes on adherence weakening over time.

We also examined the association between $\mathrm{CT}$ and adherence and found that the magnitude of the relationships between AM speech and adherence appeared to be greater (although the non-independence of AM speech and CT meant that it was not possible to test this statistically). This suggests that more attention to the theoretical basis of the relationship between change talk and behaviour change could improve understanding of the role of change talk in MI. The SDTCS offers a potentially useful tool for enhancing the MISC to incorporate SDT principles.
One area that this paper does not focus on is client language away from change or "sustain talk" (ST). Recent research has highlighted the importance of ST, and a composite variable of CT and ST has been shown to be associated with positive behaviour outcomes [7]. ST does not map directly onto the motivation continuum since $\mathrm{AM}$ and $\mathrm{CM}$ in SDT refer to different reasons to engage in a behaviour (as expressed in $\mathrm{CT}$ ) rather than reasons not to engage in a behaviour (as expressed in ST). Also, amotivation-as understood from an SDT perspective-is lacking the intention to act/acting without intent while ST refers to a desire to continue with an existing way of acting rather than making a change. Future research might focus on resolving how the concept of ST fits within the SDT theoretical framework. It might be important to consider how autonomous or controlled reasons not to engage in a behaviour or to maintain the status quo might be related to outcomes.

Although the SDTCS appears promising, further research is needed to establish its reliability and refine criteria. For example, it would be helpful to develop criteria that could further enhance inter-rater agreement alongside further refinement of the coding tool, a longer coding training requirement, and closer exploration of statements where there is a lower level of agreement.

Another potential limitation is the tool development involved the use of MI sessions with participants who rated themselves as highly motivated to change however this was deemed necessary to develop varied examples of $\mathrm{AM}$ and $\mathrm{CM}$ reasons for medication adherence. Further, it would be helpful to code AM speech and CM speech across a broader range of target behaviours. This may allow for a more nuanced understanding of client language that is currently provided by existing MI process tools (relying on the broader categories of CT) which may further add to the understanding of how MI works. Despite these limitations, this preliminary evaluation of the SDTCS suggests that it may be a useful tool for studying the process of MI and evaluating the effectiveness of MI through the lens of SDT. 
Acknowledgments The authors would like to thank the NIH for funding Project Motiv8: R01 MH068197, PI Goggin.

\section{Compliance with Ethical Standards}

Ethical Approval All procedures performed in studies involving human participants were in accordance with the ethical standards of the institutional and/or national research committee and with the 1964 Helsinki declaration and its later amendments or comparable ethical standards.

Informed Consent Informed consent was obtained from all individual participants included in the study.

Conflict of Interest The authors declare that they have no conflict of interest.

OpenAccessThis article is distributed under the terms of the Creative Commons Attribution 4.0 International License (http://creativecommons.org/ licenses/by/4.0/), which permits unrestricted use, distribution, and reproduction in any medium, provided you give appropriate credit to the original author(s) and the source, provide a link to the Creative Commons license, and indicate if changes were made.

Publisher's Note Springer Nature remains neutral with regard to jurisdictional claims in published maps and institutional affiliations.

\section{References}

1. Miller WR, Rollnick S. Motivational interviewing: helping people change. New York: Guilford press; 2012.

2. Moyers TB, Martin T, Christopher PJ, Houck JM, Tonigan JS, Amrhein PC. Client language as a mediator of motivational interviewing efficacy: where is the evidence? Alcohol Clin Exp Res. 2007;31:40s-7s.

3. Miller WR, Rose GS. Toward a theory of motivational interviewing. Am Psychol. 2009;64(6):527-37.

4. Amrhein PC. How does motivational interviewing work? What client talk reveals. J Cogn Psychother. 2004;18(4):323-36.

5. Romano M, Peters L. Understanding the process of motivational interviewing: a review of the relational and technical hypotheses. J Soc Psychother Res. 2016;26(2):220-40.

6. Copeland L, McNamara R, Kelson M, Simpson S. Mechanisms of change within motivational interviewing in relation to health behaviors outcomes: a systematic review. Patient Educ Couns. 2015;98(4):401-11.

7. Magill M, Apodaca TR, Borsari B, Gaume J, Hoadley A, Gordon $\mathrm{REF}$, et al. A meta-analysis of motivational interviewing process: technical, relational, and conditional process models of change. $\mathrm{J}$ Consult Clin Psychol. 2018;86(2):140-57.

8. Deci EL, Ryan RM. Intrinsic motivation and self-determination in human behavior. New York: Plenum Press; 1985.
9. Markland D, Ryan RM, Tobin VJ, Rollnick S. Motivational interviewing and self-determination theory. J Soc Clin Psychol. 2005;24(6):811-31.

10. Ryan RM, Plant RW, O'Malley S. Initial motivations for alcohol treatment: relations with patient characteristics, treatment involvement, and dropout. Addict Behav. 1995;20(3):279-97.

11. Williams GC, Niemiec CP, Patrick H, Ryan RM, Deci EL. The importance of supporting autonomy and perceived competence in facilitating long-term tobacco abstinence. Ann Behav Med Publ Soc Behav Med. 2009;37(3):315-24.

12. Williams GC, Rodin GC, Ryan RM, Grolnick WS, Deci EL. Autonomous regulation and long-term medication adherence in adult outpatients. Health Psychol Off J Div Health Psychol Am Psychol Assoc. 1998;17(3):269-76.

13. Kennedy S, Goggin K, Nollen N. Adherence to HIV medications: utility of the theory of self-determination. Cogn Ther Res. 2004;28(5):611-28.

14. Houck JM, Moyers TB, Miller WR, Glynn L, Hallgren K. Motivational interviewing skill code (MISC) Version 2.5. [Internet]. 2010. Available from: http://casaa.unm.edu/download/ misc25.pdf

15. Williams GC, McGregor HA, Zeldman A, Freedman ZR, Deci EL. Testing a self-determination theory process model for promoting glycemic control through diabetes self-management. Health Psychol Off J Div Health Psychol Am Psychol Assoc. 2004;23(1):58-66.

16. Brod M, Tesler LE, Christensen TL. Qualitative research and content validity: developing best practices based on science and experience. Qual Life Res. 2009;18(9):1263-78.

17. Resnicow K, McMaster F. Motivational interviewing: moving from why to how with autonomy support. Int $\mathrm{J}$ Behav Nutr Phys Act. 2012;9:19.

18. Goggin K, Gerkovich MM, Williams KB, Banderas JW, Catley D, Berkley-Patton $\mathrm{J}$, et al. A randomized controlled trial examining the efficacy of motivational counseling with observed therapy for antiretroviral therapy adherence. AIDS Behav. 2013;17(6):1992-2001.

19. Moyers TB, Martin T. Therapist influence on client language during motivational interviewing sessions. J Subst Abus Treat. 2006;30(3): 245-51.

20. Cohen J. A coefficient of agreement for nominal scales. Educ Psychol Meas. 1960;20(1):37-46.

21. Gwet KL. Computing inter-rater reliability and its variance in the presence of high agreement. Br J Math Stat Psychol. 2008;61(1): 29-48.

22. Wongpakaran N, Wongpakaran T, Wedding D, Gwet KL. A comparison of Cohen's Kappa and Gwet's AC1 when calculating interrater reliability coefficients: a study conducted with personality disorder samples. BMC Med Res Methodol. 2013;13(1):61.

23. Lee IA, Preacher KJ. Calculation for the test of the difference between two dependent correlations with one variable in common [Internet]. 2013. Available from: http://quantpsy.org.

24. Lord SP, Can D, Yi M, Marin R, Dunn CW, Imel ZE, et al. Advancing methods for reliably assessing motivational interviewing fidelity using the motivational interviewing skills code. J Subst Abus Treat. 2015;49:50-7. 